\title{
Effect of Bite with and without Dentures on Soleus H-Reflex in the Elderly
}

\author{
KAZUNORI MOROZUMI $^{1)}$, TAKUYA NISHIYA ${ }^{1)}$, AKIMASA YASUDA ${ }^{1)}$, YUKIO OIDA ${ }^{2)}$, \\ TAKAYUKI FUJIWARA ${ }^{3)}$, CHENG GOH AH ${ }^{3}$, IWAO YAMAMOTO ${ }^{4)}$ \\ 1) Department of Rehabilitation, Hachiohji Health Cooperative Shiroyama Hospital: \\ 3-2872-1 Motohachiohjimachi, Hachiohji, Tokyo 193-0826, Japan. TEL +81 426-65-2611 \\ 2) Meiji Physical Fitness Research Institute \\ ${ }^{3)}$ Shinshu University School of Allied Medical Sciences \\ 4) Department of Functional Polymer Science, Faculty of Textile Science and Technology, \\ Shinshu University
}

\begin{abstract}
We reported previously on the necessity of the elderly wearing dentures while they take physical therapy, because of improved maximum muscular strength and agility under biting with dentures compared to without dentures. We assumed that this phenomenon resulted from improved strength of the masseter muscle and occlusal force brought about by wearing dentures. In order to corroborate our assumption, we selected eight elderly subjects (age: 71-98; mean age: 83.9) and measured the soleus H (Hoffman wave) reflex as an index of excitability in the spinal cord motor neuron pool, activity of the masseter muscle, and occlusal force of incisors under biting with and without dentures, so as to examine changes in the measured parameters. The results were that the values measured with dentures were significantly greater than those without dentures. We consider that these data support our assumption.
\end{abstract}

Key words: H-reflex, Elderly, Dentures.

(This article was submitted Oct. 2, 2001, and was accepted Feb. 25, 2002)

\section{INTRODUCTION}

Average life expectancy has shown a rapid increase in Japan, and our country is ranked first as a nation of longevity ${ }^{1)}$. However, it is not also true of the average life of teeth ${ }^{2}$, and most elderly persons, both healthy and disabled, cannot get along without a full set of dentures.

We previously tested how the presence and absence of dentures in the elderly influenced their motor performance ${ }^{3)}$. We selected two test methods that are safe and simple for the elderly to measure maximum muscular strength, agility, flexibility and balance, all of which tend to decline with age ${ }^{4)}$. The first was the grip strength test for determining muscular strength, and the other was the bar gripping test for determining bar-gripping reaction time $^{5)}$, that is used as a means for discriminating between the central and the peripheral processes participating in kinetic expression. Superior results were obtained both in the maximum grip strength and in the bar-gripping reaction time when measured under biting with dentures compared to those without dentures. Based on these data, we suggested the necessity of wearing dentures for the elderly when taking physical therapy. We considered that the increased strength and shortened reaction time were induced by a lift of the occlusal position (an occlusion with the mouth opening slightly relative to the normal centric occlusal position) in the presence of dentures increasing the tension of the masseter muscle, thereby producing a higher occlusal force, which influenced clenching ${ }^{6-8)}$ during the maximum physical exercise. 


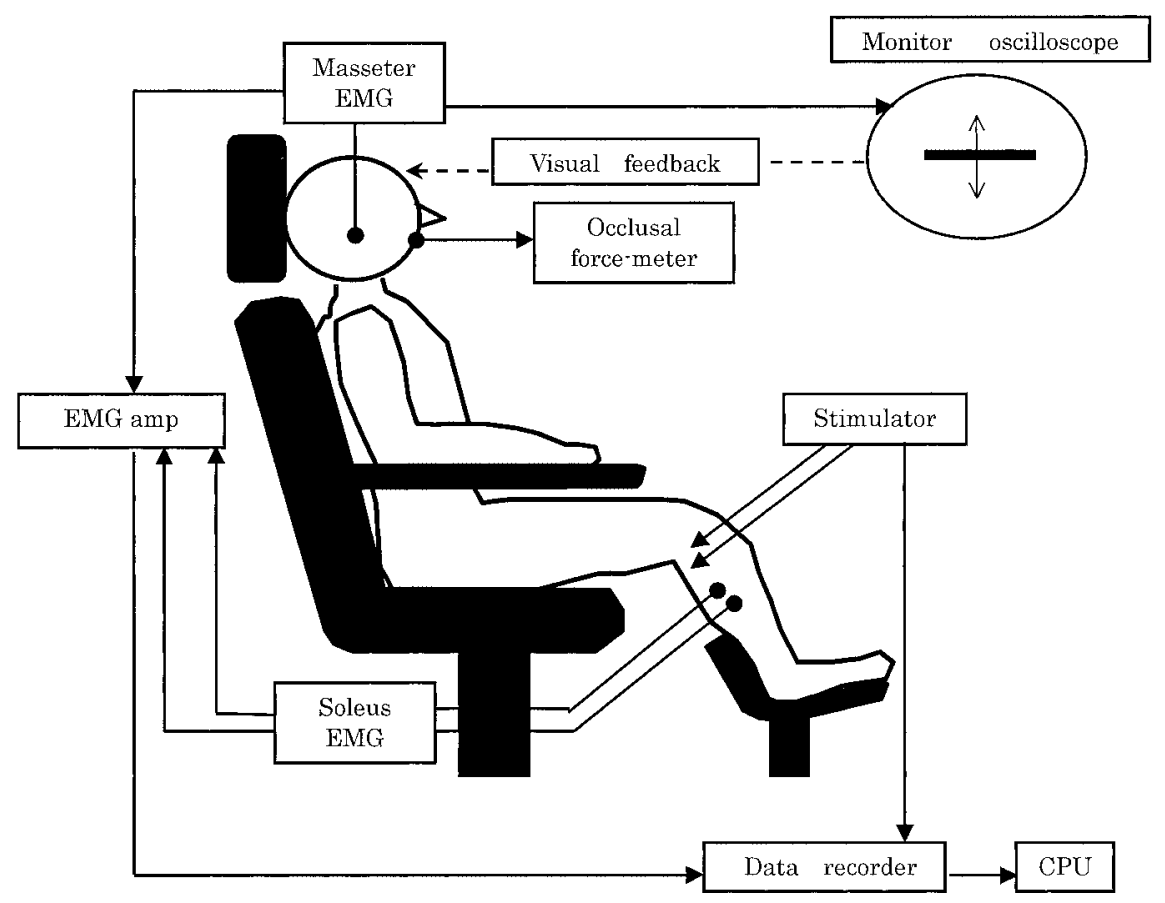

Fig. 1. Block diagram of experiment.

However, we conducted neither determination of the occlusal force nor observation of activity of the masseter and facial muscles, so we were unable to elucidate the mechanism by wich the change in occlusal force when wearing dentures influenced the grip strength and bar-gripping reaction time.

The purpose of this study was to elucidate this mechanism. We measured the soleus H (Hoffman wave) reflex ${ }^{9-15)}$, which indicates the excitability in the spinal cord motor neuron pool, activity of the masseter muscle and occlusal force of incisors, under biting in the presence and absence of dentures, to examine the changes in the measured values.

\section{METHODS}

Eight healthy subjects (male: 4; female: 4) were selected. They were aged between 71 and 98 (mean age: 83.9 ) and had a height of 125 to $168.6 \mathrm{~cm}$ (mean height: $148.6 \mathrm{~cm}$ ) and a body weight of 35 to $66 \mathrm{~kg}$ (mean weight: $52.2 \mathrm{~kg}$ ).

\section{Procedures}

Each subject was seated in a relaxed position in a reclining chair with a knee flexion angle of $40 \mathrm{deg}$. and an ankle plantar flexion angle of $20 \mathrm{deg}$. In this state, the soleus H-reflex, activity of the masseter muscle and occlusal force of incisors were measured under the following four conditions: 1) open mouth with dentures; 2) maximum biting with dentures; 3) open mouth without dentures; and 4) maximum biting without dentures. A monitor was set in front of the subject at a distance of about $1 \mathrm{~m}$ (see Fig. 1), and the subject was directed to clench hard and to open the mouth slightly so that the subject could assume the maximum voluntary occlusion and the open-mouth posture by monitoring a waveform of integrated EMG in the masseter muscle displayed on the unit.

\section{(1) Soleus H-reflex}

The soleus H-reflex was evoked by stimulating the tibial nerve on the popliteal fossa of each subject seated in a reclining chair in a relaxed position with a constant current $(1 \mathrm{msec}$. duration rectangular wave). The size of the control H-reflex under open mouth was adjusted to about $30 \%$ of $\mathrm{M}_{\max }$. The peak-to-bottom amplitude of H-reflex was measured five times at random intervals under each condition using a signal processor (DP-1200, NEC Medical Systems) at a sampling frequency of 1 
Table 1. Mean value of each test

\begin{tabular}{llccc}
\hline & & Without Dentures & t-test & With Dentures \\
\hline H-Reflex open & $\mathrm{mv}$ & $2.98 \pm 2.52$ & $\mathrm{~ns}$ & $2.93 \pm 2.5$ \\
H-Reflex bite & $\mathrm{mv}$ & $3.64 \pm 2.96$ & $*$ & $4.13 \pm 3.34$ \\
H-Reflex ratio (bite/open) & $\%$ & $28.8 \pm 41$ & $*$ & $59.5 \pm 54$ \\
Integrated masseter muscle & $\mu \mathrm{v} \cdot \mathrm{sec}$ & $44.7 \pm 31$ & $*$ & $95.8 \pm 74$ \\
Occlusal force & $\mathrm{kg}$ & $0.86 \pm 1.6$ & $* *$ & $4.29 \pm 2.1$ \\
\hline
\end{tabular}

$\mathrm{ns}=$ not significant. ${ }^{*} \mathrm{p}<0.05$, paired t-test. ${ }^{* *} \mathrm{p}<0.01$, paired t-test

$\mathrm{KHz}$. The measured H-reflex values were averaged out to calculate the increase ratio of the mean soleus H-reflex under biting to that under open mouth with and without dentures, respectively, and the resulting data were analyzed by the paired t-test.

\section{(2) Activity of the masseter muscle}

Activity of the masseter muscle was recorded through surface electrodes $(\mathrm{Ag}-\mathrm{AgCl})$ applied to the belly of the masseter muscle at an electrode-toelectrode distance of $3 \mathrm{~cm}$ (cutaneous impedance was preset to $5 \mathrm{k} \Omega$ or less, at 20 to $500 \mathrm{~Hz}$ ). The activity during maximum biting was determined five times each in the presence and absence of dentures by full wave rectification and integration in the period $500 \mathrm{msec}$ immediately before the rising edge of the evoking stimulation. Mean values of discharge integral in the masseter muscle, which were obtained in the five trials, were calculated respectively and were analyzed by the paired t-test.

\section{(3) Occlusal Force of Incisors}

After completion of H-reflex measurement, the maximum occlusal force of incisors was measured twice with and without dentures by an occlusal force meter GM10 (Nagano Keiki). Mean values were calculated and were analyzed by the paired ttest.

\section{RESULTS}

\section{H-Reflex}

The mean H-reflex values were significantly greater under biting with dentures $(p<0.05$, paired $t$ test) compared with those without dentures. The Hreflex increased by $59.5 \%$ under biting with dentures and by $28.8 \%$ under biting without dentures, on average, compared with those under open mouth conditions (Table 1, Fig. 2).

\section{Activity of Masseter Muscle}

Activity of the masseter muscle under maximum biting with dentures was significantly greater $(p<0.05$, paired $t$-test) than that without dentures (Table 1).

\section{Occlusal Force of Incisors}

The occlusal force of incisors measured with dentures was significantly greater $(\mathrm{p}<0.01$, paired $\mathrm{t}-$ test) than that measured without dentures (Table 1).

\section{FINDINGS}

We assumed, as a result of our previous study, that the elderly show increased occlusal forces when they wear dentures properly, due to a lift of the occlusal position which enhances tension of the masseter muscle allowing it to work efficiently, thereby influencing clenching during maximum physical exercise ${ }^{6-8)}$, and in turn, the maximum muscular strength and agility. The results obtained in this study showed a significantly improved soleus $\mathrm{H}$-reflex increase ratio, activity of the masseter muscle and occlusal force in the presence of dentures. We believe that these data support our original assumption.

"Influence of Wearing Dentures on Strength of Masseter and Incisor Occlusal Force"

We believe that dentures influence the occlusal position and increase tension of the masseter muscle, inducing high activity of the masseter muscle. Ishigami ${ }^{8)}$ reports that a lift of the occlusal position (an occlusion with the mouth open slightly relative to the normal centric occlusal position) caused by wearing a mouth piece increases tension of the masseter muscle and that the teeth of the upper jaw are respectively brought into contact with those of the lower jaw evenly at the centrical occlusal position (reference positions of the teeth of 

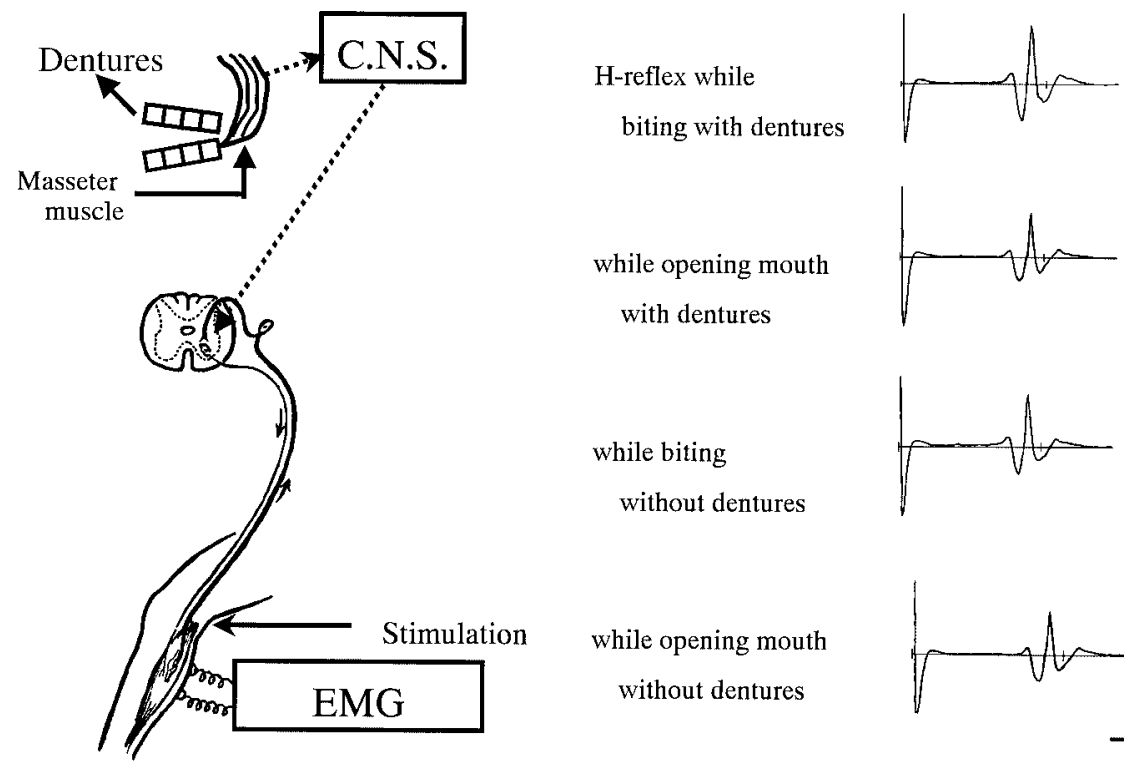

while opening mouth

without dentures

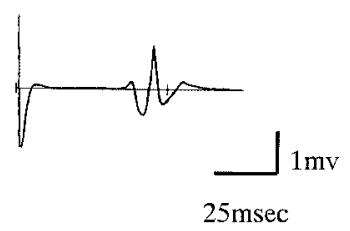

Fig. 2. Schematic illustration of H-reflex with data sample.

upper and lower jaws), favorably influencing motor performance. In view of this report, we consider that the lift of the occlusal position caused by the presence of dentures increased the activity of the masseter muscle and that the increased strength of the masseter muscle produced a higher occlusal force of the incisors.

\section{"Influence of Wearing Denture on H-reflex"}

We consider that the presence of dentures influences the occlusal position, increasing tension of the masseter muscle, and produces a strong occlusal force thereby inducing a high H-reflex amplitude. This means the increase of progressive stimulation of the spinal cord motor neuron on the reflex pathway of the $\mathrm{H}$-wave. Miyahara et al. ${ }^{14,15)}$ explained these phenomena: A descending input from the superior central nerve and the sensory information from the peripheral nerve to the spinal cord monosynaptic reflex arc influence facilitation of soleus H-reflex at the time of clenching, like in the maneuver of Jendrassik's ${ }^{16-18)}$ who analyzed facilitation dynamics of H-reflex at the time of voluntary contraction of muscles in the remote parts of the body such as the antebrachial muscle. Cowan et al. ${ }^{19)}$ analyzed the time-related effect of percutaneous motor cortex stimulation on H-reflex facilitation. Their results suggested monosynaptic transmission of stimulation from the corticospinal tract to the motor neuron. Takada ${ }^{20)}$ and Hagiya ${ }^{21)}$ analyzed the effects of occlusion and rhythmical jaw movement on the monosynaptic reflexes of the extensor and flexor of the ankle, and both groups of researchers reported that the monosynaptic reflexes were facilitated nonreciprocally and that excitatory input from the peripheral and superior central nerves influenced facilitation of the H-reflex. We consider that the presence of dentures influenced the activity of the masseter muscle and also the occlusal force of incisors, and in turn, the H-reflex.

In this study, we tested how the soleus H-reflex, activity of the masseter muscle and occlusal force are influenced by the presence and absence of dentures. Soleus H-reflex, activity of the masseter muscle and occlusal force all showed improving tendencies in the presence of dentures. These results suggest that the presence and absence of dentures may influence the motor performance in the elderly when they take physical therapy.

It will be necessary for us to clarify further the mechanism of the influence of increased activity of the masseter muscle and increased occlusal force upon motor performance.

\section{REFERENCES}

1) Fujii Y: Suggestions (50): Infant mortality - as 
barometer of average life expectancy in each country of the world, in terms of Japanese fiscal yearCorrection based on the United Nations' dynamics of population 1993. J Pharm, 1996, 32 (11): 129-138 (in Japanese).

2) Institute of Health Care Association, Ministry of Health and Welfare: Report on Actual Condition Survey of Dental Diseases, 1989, pp 29-31 (in Japanese).

3) Morozumi K, Imai H, Nishiya $T$, et al.: Influence of dentures worn by the elderly on grip strength and reaction time. Rigaku ryohogaku, 1999, 26 (1): 14-16 (in Japanese).

4) Ishikawa T: Evaluation of physical strength in old age. Science of Physical Capacity, 1993, 42: 16-18 (in Japanese).

5) Iwatuki H, Muroga T: Age-related change in manual reaction time. Exercise Physiology, 1990, 5 (2): 65-69 (in Japanese).

6) Ichioka $\mathrm{N}$, Koshino $\mathrm{T}$, Ishijima $\mathrm{T}$, Tanaka $\mathrm{S}$, Hirai $\mathrm{T}$ : Influence of change in underjaw position on clenching strength and general motor performance. Supplement, 1992, 36 (Special Issue 87): 123 (in Japanese).

7) Ishijima $T$, Hirai $T$, Imamura M: Study on frequency of clenching occurring during general physical exercise. Supplement, 1991, 35: 193-199 (in Japanese).

8) Ishigami K: Outline of sport dentistry. Science of Physical Capacity, 1996, 45: 531-540 (in Japanese).

9) Fujiwara $T$, Ishikawa $T$ : Change in H-reflex during maneuver of facilitation of nerve. Sogo Rehabilitation, 1982, 10: 1009-1014 (in Japanese).

10) Fujiwara $T$, Ishikawa $T$, Miyazawa $T$ : Study of changes of progressive stimulation in spinal cord. Science of Physical Capacity, 1980, 29: 237 (in Japanese).

11) Yanagisawa $K$, Nakamura R, Fujiwara T: Influence of arm PNF position on soleus H- reflex. Rigaku ryohogaku, 1989, 16: 19-22 (in Japanese).

12) Yanagisawa K, Ohhashi $Y$, Saitou H, Nakamura R,
Fujiwara T: Influence of leg PNF position on counterlateral soleus H-reflex. Rigaku ryohogaku, 1990, 17 (Special Issue prepared for Academic Conference): 75 (in Japanese).

13) Ohyama T, Miyahara T, Suzuki R, Shinozuka O, Nakamura K: Occlusion and competing force. J Japan Dental Association, 1991, 43 (10): 11-18 (in Japanese).

14) Miyahara $T$ : Disorder in human soleus H-reflex Induced by clenching. J Stomatology, 1991, 58 (4): 670-686 (in Japanese).

15) Miyahara $T$, Hagiya $N$, Ohyama $T$, Nakamura $Y$ : Modulation of human soleus H-reflex in association with voluntary clenching of the teeth. J Neurophysiol, 1996, 76 (3): 2033-2041.

16) Delwaide PJ, Toulouse P: Jendrassik maneuver vs controlled contractions the excitability of soleus monosynaptic reflexes. Arch Phys Med Rehabil, 1980, 61 (November): 505-510.

17) Delwaide PJ, Toulouse P: Facilitation of monosynaptic reflexes by voluntary contraction of muscles in remote parts of the body. Brain, 1981, 104: 701-719.

18) Delwaide PJ, Toulouse P: The Jendrassik maneuver/ quantitative analysis of reflex reinforcement by remote voluntary muscle contraction. Motor Control Mechanisms in Health and Disease, 1983, 661-669.

19) Cowan JMA, Day BL, Marsden C, Rothwell JC: The effect of percutaneous motor cortex stimulation on $\mathrm{H}$ reflexes in muscles of the arm and leg in intact man. $\mathrm{J}$ Physiol, 1986, 377: 333-347.

20) Takada N: Facilitation of H-reflexes in human anterior tibial muscle in association with clenching and attenuation of reciprocal Ia inhibition against soleus. J Oral Diseases, 1998, 65 (1): 64-73 (in Japanese).

21) Hagiya N: Modulation of spinal cord monosynaptic reflex in association with rhythmical jaw movement and neuromechanism. J Oral Diseases, 1994, 61 (1): 21-38 (in Japanese). 